\title{
GAMMA-RAY BURSTS: THE DEPENDENCE OF THE SPECTRAL LAG ON THE ENERGY
}

\author{
P. Minaev ${ }^{1}$, A. Pozanenko ${ }^{1}$, S. Grebenev ${ }^{1}$ and S. Molkov ${ }^{1}$
}

\begin{abstract}
We investigated the dependence of a spectral lag against energy band based on 28 bright GRBs registered by SPI and IBIS/ISGRI of INTEGRAL observatory. It was found that for simple structure bursts or well separated pulses of multipulse bursts the spectral lag can be approximated by the relation of $\tau \sim A \lg (E)$, where $\mathrm{A}$ is a positive parameter, which correlates with pulse duration. We have not found any negative lag in simple structure bursts or in well separated pulses. While investigating the time profile of the whole burst negative lag may appear due to different spectral parameters of the pulses.
\end{abstract}

\section{Introduction}

Spectral evolution of gamma-ray bursts is one of the most interesting phenomenological properties. In most cases, we observe the evolution from the hard spectrum in the beginning, to soft, in the final phase of gamma-ray burst. Different dependencies between the spectral evolution and other properties of gamma-ray bursts have been found (Hakkila et al. 2011). One of the models describing spectral evolution of GRBs is based on curvature effect of the relativistic shocked shell (Ukwatta et al. 2012).

\section{Data analysis and results}

28 bright GRBs registered by SPI (Vedrenne et al. 2003) and IBIS/ISGRI (Ubertini et al. 2003) experiments of INTEGRAL observatory (Winkler et al. 2003) were investigated. Well separated pulses of multipulse events were investigated independently. So total number of analyzed events is 43. Method of investigation was based on cross-correlation analysis of light curves in two different energy bands. Details of cross-correlation analysis see in (Band 1997). In this analysis

\footnotetext{
1 Space Research Institute of the Russian Academy of Sciences, Moscow, Russia; e-mail: minaevp@mail.ru
} 
cross-correlation function $(\mathrm{CCF})$ of two light curves in different energy channels is formed. Position of the maximum in CCF curve determines the value of light curves time offset, which is called spectral lag. Spectral lag is positive when light curve in higher energy band is registered earlier than one in lower energy band.

Algorithm of our analysis consisted of next steps:

1. Building a GRB energy-time diagram (Fig. 1a) and visual analysis of GRB spectral and temporal properties (hardness of GRB spectrum, number of pulses, duration, etc.).

2. Building a light curves in narrow energy bands (up to 25 channels) and selection of time interval for analysis. Time resolution and energy channel width of light curves depend only on GRB properties.

3. Cross-correlation analysis of formed light curves to determine spectral lag between light curve in the first lowest energy channel and light curves in other channels.

4. Forming and approximation of spectral lag - energy relation (Fig. 1c) using two models (formulas 2.1-2.2), where parameter A (spectral lag index) characterizes spectral evolution. Energy value of each point of relation was defined as geometric mean of upper and lower energy limit of the energy channel in higher energy band.

$$
\begin{gathered}
\tau=A \log (E)+B . \\
\tau=\left(A_{1} \log (E)+B_{1}\right) \exp \left[\left[-\frac{\log (E)}{\log \left(E_{\text {cut }}\right)}\right]^{C}\right]+ \\
+\left(A_{2} \log (E)+B_{2}\right)\left[1-\exp \left[\left[-\frac{\log (E)}{\log \left(E_{\text {cut }}\right)}\right]^{C}\right]\right] ; \\
B_{2}=E_{\text {cut }}\left(A_{1}-A_{2}\right)+B_{1} .
\end{gathered}
$$

In most cases lag - energy relation is well described by simple logarithmic model (2.1) (Fig. 1c, left). Positive slope of the relation means spectral evolution from hard spectrum to soft (positive value of spectral lag). But in 6 cases of 43 in the relation there is break (Fig. 1c, right). In this 6 cases two-logarithmic model with exponential break (2.2) was used to fit the relation.

Spectral lag - energy relation for separate pulses of GRBs or for GRBs with simple structure of light curves (Fig. 1b, left) shows no break (Fig. 1c, left) and no negative lag. Break in the relation and negative lag may appear due to different spectral parameters of the overlapping pulses and have no connection with physics of the GRB source.

For 9 well separated GRB pulses spectral lag index - duration relation was formed. The relation is well fitted by power law with power index equal to 

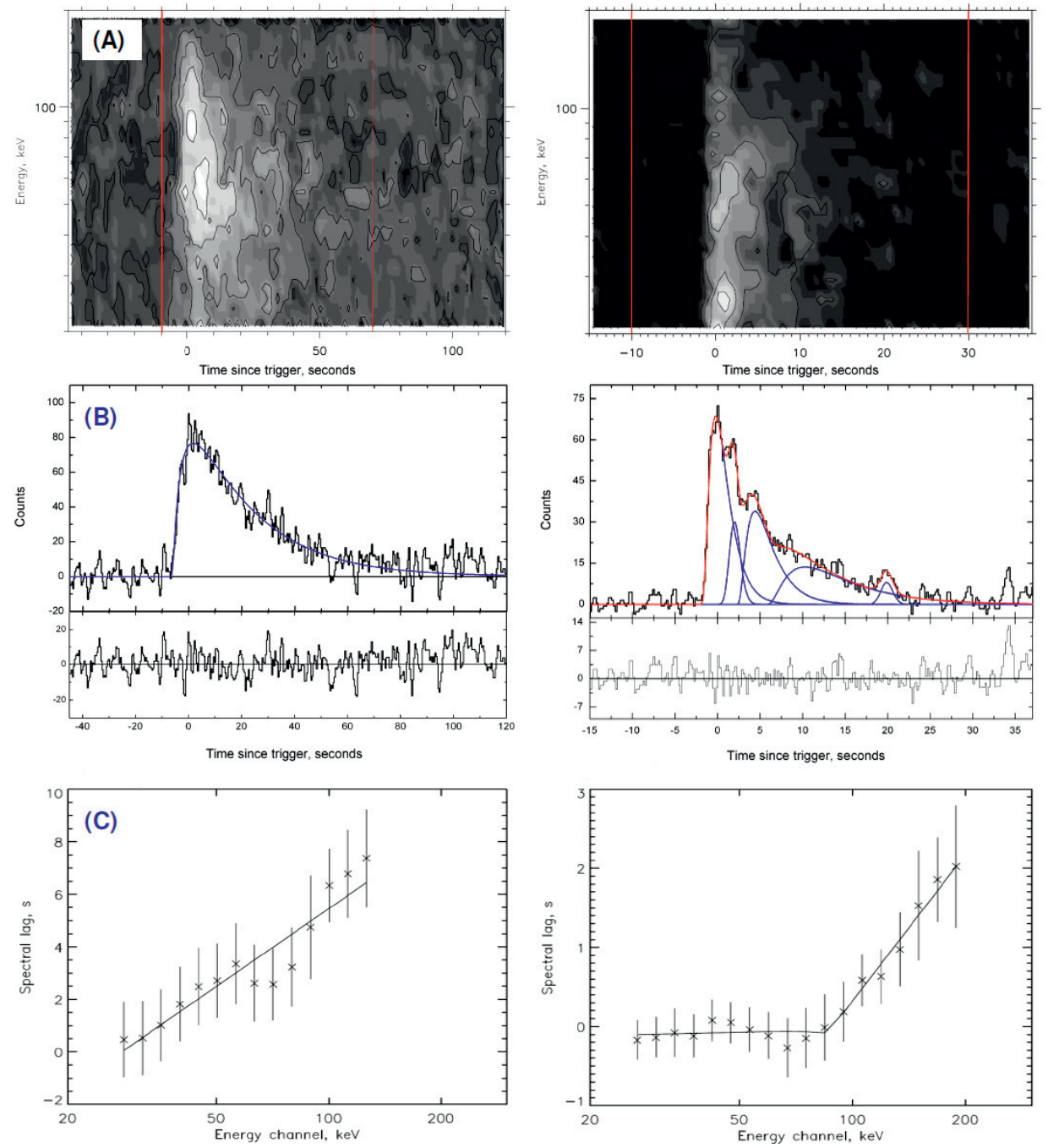

Fig. 1. Energy - time diagram (A), mask weighted light curve in [20-200] keV energy band (B) and spectral lag - energy relation (C) of GRB 050504 (left) and GRB 031203 (right).

$1.14 \pm 0.15$. There is one short GRB 081226 in the sample and it does not violate the correlation. So short GRBs may follow the same law as the long ones and it may be the evidence of the same emission mechanism in short and long GRBs. This correlation was also found in paper (Hakkila et al. 2011). But in that paper spectral lag between light curves in BATSE energy channels was used as spectral evolution parameter instead of spectral lag index. 


\section{Conclusions}

Spectral evolution of 28 bright GRBs registered by SPI and IBIS/ISGRI of INTEGRAL observatory was investigated.

It was found that for simple structure bursts or well separated pulses of multipulse bursts the spectral lag can be approximated by the simple logarithmic law (2.1).

Parameter A (spectral lag index) is new alternative parameter characterizing spectral evolution of GRBs.

Spectral lag index correlates with pulse duration. The dependence of the spectral lag index on the duration of GRB pulses is presumably the same for long and for short GRBs.

Negative lag in simple structure bursts or in well separated pulses was not found.

The work was partially supported by RFBR grant 12-02-01336-a.

\section{References}

Band, D., 1997, ApJ, 486, 928

Hakkila, J., \& Preece, R., 2011, ApJ, 740, 104

Ubertini, P., Lebrun, F., Di Cocco, G., et al., 2003, A\&A, 411, L131

Ukwatta, T., Dhuga, K., Stamatikos, M., et al., 2012, MNRAS, 419, 614

Vedrenne, G., Roques, J.-P., Schonfelder, V., et al., 2003, A\&A, 411, L63

Winkler, C., Courvoisier, T., Di Cocco, G., et al., 2003, A\&A, 411, L1-L6 